\title{
Analytic inversion of a conical Radon transform arising in application of Compton cameras on the cylinder
}

\author{
Sunghwan Moon ${ }^{\dagger} \quad$ Markus Haltmeier ${ }^{\ddagger}$ \\ †Department of Mathematical Sciences, Ulsan National Institute of Science and Technology \\ Ulsan 44919, Republic of Korea. \\ shmoon@unist.ac.kr \\ ${ }_{\ddagger}^{\ddagger}$ Department of Mathematics, University of Innsbruck \\ Technikerstrasse 13, A-6020 Innsbruck, Austria \\ Markus.Haltmeier@uibk.ac .at
}

\begin{abstract}
Single photon emission computed tomography (SPECT) is a well established clinical tool for functional imaging. A limitation of current SPECT systems is the use of mechanical collimation, where only a small fraction of the emitted photons is actually used for image reconstruction. This results in large noise level and finally in a limited spatial resolution. In order to decrease the noise level and to increase the imaging resolution, Compton cameras have been proposed as an alternative to mechanical collimators. Image reconstruction in SPECT with Compton cameras yields to the problem of recovering a marker distribution from integrals over conical surfaces. Due to this and other applications, such conical Radon transforms recently got significant attention. In the current paper we consider the case where the cones of integration have vertices on a circular cylinder and axis pointing to the symmetry axis of the cylinder. As main results we derive analytic reconstruction methods for the considered transform. We also investigate the $\mathrm{V}$-line transform with vertices on a circle and symmetry axis orthogonal to the circle, which arises in the special case where the absorber distribution is located in a horizontal plane.
\end{abstract}

Keywords: Conical Radon transform, nuclear imaging, Compton cameras, SPECT, image reconstruction, inversion formula

AMS Subject Classification: 44A12, 65R10, 92C55.

\section{Introduction}

In this paper we study the inversion of a conical Radon transform that maps a function defined in three dimensional space to its integrals over a special family of cones. Recovering a function from integrals over cones arises in SPECT using Compton cameras. These type 
measurement devices have been introduced as an alternative to classical gamma cameras based on mechanical collimators with increased sensitivity [6, 19, 23]. Inversion of conical Radon transforms is also relevant for single scattering optical tomography [7] or Compton scattering imaging [15]. Recently various versions the conical Radon transforms have been studied (see for example [1, 3, 5, 8, 9, 12, 14, 15, 18, 20, 21, 22, and the references therein). The instance of the conical Radon transform that we study in this paper arises in application of SPECT using a cylindrical Compton camera.

\subsection{Compton cameras in SPECT}

In SPECT, weakly radioactive tracers are given to the patient and participate in physiological processes. The radioactive tracers can be detected through the emission of gamma ray photons allowing to infer information about physiological processes. In order to obtain sufficient location information about the emitted photons, the standard approach in SPECT is to use collimators, which only record photons that enter the detector surface vertically. As illustrated in the left image in Figure1.1, such data provide integrals of the tracer distribution over straight lines, and reconstructing the tracer distribution can be performed by inverting the (attenuated) ray transform [25]. A main drawback of the use of mechanical collimators is that they remove most photons, and therefore the number of recorded photons is low. To increase the sensitivity, the use of Compton cameras has been proposed in [6, 19, 23]. Opposed to classical gamma cameras, Compton cameras use two detector arrays in order to avoid the use of a mechanical collimator.

As illustrated in the right image in Figure 1.1, a Compton camera consists of a scatter detector array $D_{s}$ and an absorption detector array $D_{a}$. A photon emitted in the direction of the camera undergoes Compton scattering in $D_{s}$, and is absorbed in $D_{a}$. In each detector, the position and the energy of the photon are measured [19]. The measured energies can be used to determine the scattering angle or half opening angle $\psi$ at $D_{s}$ via the Compton scattering formula $\cos (\psi)=1-m c^{2}\left(E_{s}-E_{a}\right) /\left(E_{s} E_{a}\right)$, where $m$ is the electron mass, $c$ the speed of light, $E_{s}$ the photon energy at $D_{s}$, and $E_{a}$ the energy of the photon measured at $D_{a}$. Using this information, one can conclude that the observed photon must have been emitted on the surface of a circular cone, where the vertex is given by the position $x_{s}$ at $D_{s}$, the central axis points from $x_{a}$ (the position at $D_{a}$ ) to $x_{s}$, and the scattering angle is given by $\psi$.

Now suppose we have given a distribution of traces $f: \mathbb{R}^{3} \rightarrow \mathbb{R}$ which emit photons uniformly in all directions. The expected number of photons recorded with data $\left(x_{s}, E_{s}\right)$ and $\left(x_{a}, E_{a}\right)$ is proportional to $K(\psi) I$, where $I$ is the integral over the cone determined by $\left(x_{s}, E_{s}\right)$ and $\left(x_{a}, E_{a}\right)$ and $K(\psi)$ is the Klein-Nishina distribution which describes the probability that a given photon scatters by angle $\psi$. The Klein-Nishina distribution is known explicitly and well bounded from below for typical photon energies. After rescaling we can therefore assume that Compton cameras provide noisy versions of $I$, from which the tracer distribution $f$ has to be recovered. 

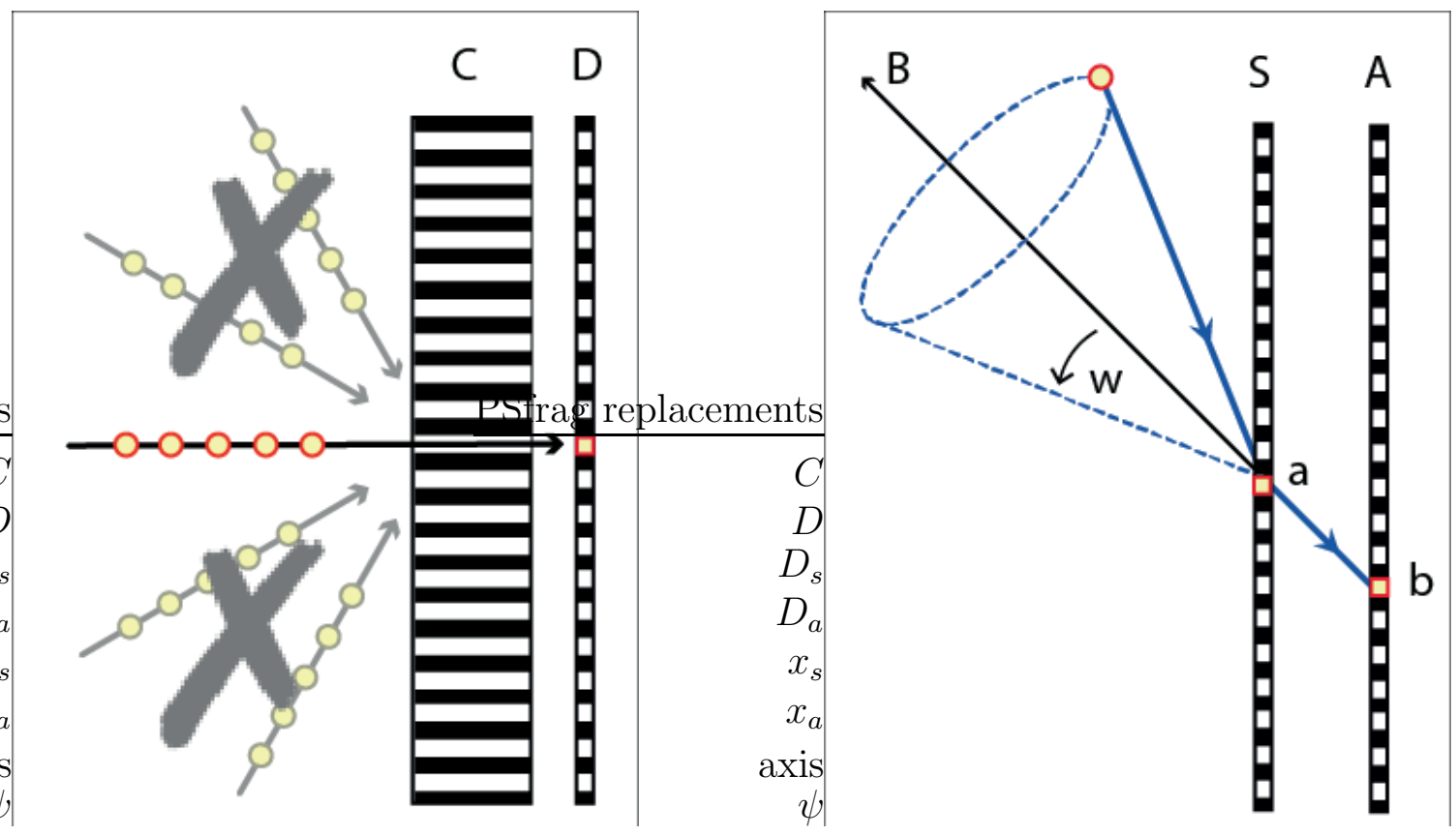

Figure 1.1: Collimator versus Compton CAMERA. Left: In standard gamma cameras, a collimator $C$ is inserted, which only observes photons propagating orthogonal to the detector plane. The location of any emitted photon can be traced back to a straight line. Right: A Compton camera consists of two detector arrays $D_{s}$ and $D_{a}$. Every observed photon can be traced back to the surface of a cone.

\subsection{The conical Radon transform on the cylinder}

In this work we consider the situation where the scattering detector forms a cylindrical surface $\mathbb{S}^{1} \times \mathbb{R}$ and that the source distribution $f$ is assumed to be supported inside this cylinder. We assume that the available data consist of integrals over all cones with the vertex on $\mathbb{S}^{1} \times \mathbb{R}$ and the central axis pointing to $\{(0,0)\} \times \mathbb{R}$; see Figure 1.2 (a). Our goal is to recover $f$ from its conical Radon transform, consisting of integrals of $f$ over these cones. One possible way to realize a cylindrical Compton camera is illustrated in Figure 1.2 (b), where, in a first step, data are collected with a one-dimensional Compton camera [2, 13. Such a onedimensional Compton camera consists of two linear detector arrays and records integrals over cones with axis intersecting both linear detectors. In order to obtain the considered data, the one-dimensional Compton camera is rotated around the $x_{3}$-axis. In [13] it has been shown that data of a one-dimensional Compton camera without rotation is theoretically (almost) sufficient to recover the absorber distribution. While $f$ has 3 -dimensional domain, the class of cones we consider depends on four variables, and so we are facing an overdetermined inverse problem. Due to low photon counts the use of such over-determinated data is actually a desired feature of Compton cameras (see, for example, the discussion in [1]).

Additionally, we consider a special two-dimensional version of the conical Radon transform. Suppose that the support of the phantom is thin and contained in a horizontal plane, such that we can model it by $f\left(x_{1}, x_{2}, x_{3}\right)=\delta\left(x_{3}\right) F\left(x_{1}, x_{2}\right)$, where $\delta$ is the one-dimensional 


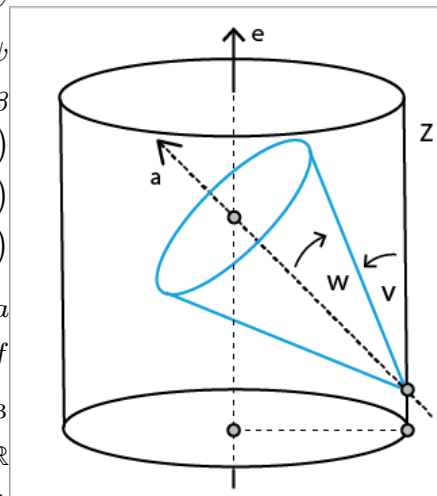

A

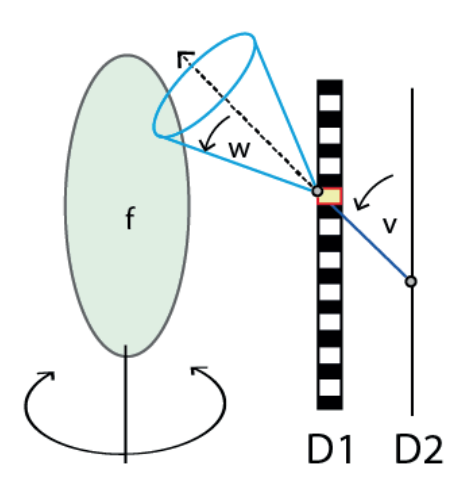

B

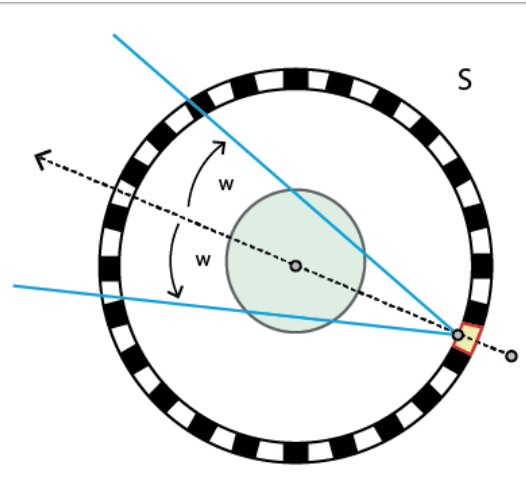

C

Figure 1.2: Conical RAdON TRAnsform ON THE CYlinder. (a) The conical Radon transform integrates a function over all cones with the vertex on $\mathbb{S}^{1} \times \mathbb{R}$ and the axis intersecting $\{(0,0)\} \times \mathbb{R}$. (b) Vertical cross section. The conical integrals may be obtained by rotating a one-dimensional Compton camera rotated around the $e_{3}$-axis. (c) Horizontal cross section. The conical integrals reduce to integrals over V-shaped, with the vertex on $\mathbb{S}^{1}$ and the symmetry axis pointing to the origin.

delta-distribution. In such a situation it is reasonable to restrict the set of vertices to $\mathbb{S}^{1} \times\{0\}$. The intersection of any corresponding cone with the plane $\left\{x \in \mathbb{R}^{3} \mid x_{3}=0\right\}$ becomes a $\mathrm{V}$-line with the vertex on $\mathbb{S}^{1}$ and the axis pointing to the origin; see Figure 1.2 (c). Reconstructing the function $F$ from the resulting $\mathrm{V}$-line transform will be considered in Section 2. In fact the developed inversion method for the $\mathrm{V}$-line transform is the basis of one of our inversion methods for the three-dimensional conical Radon transform. Another interesting two-dimensional case would arise when $f$ is restricted to a vertical plane and the vertices of the cones are located on a line pointing in the $x_{3}$-direction. The resulting $\mathrm{V}$-line transform with vertices on a line been studied in previous works, such as [1, 2, 12, 9, 24, and therefore will not be investigated in the present work.

\subsection{Outline}

This manuscript is organized as follows. In Section 2 we define the V-line transform with vertices on the circle and derive an explicit inversion formula based on a Fourier series expansion. This in particular implies the invertibility of the V-line transform. In Section 3 we present two inversion methods for inverting the conical Radon transform with vertices on the cylinder and the axis pointing to the symmetry axis of the cylinder. The first method even works when a radial weight included in the conical Radon transform and is based on reducing the conical Radon transform to the V-line transform. The second method uses an approach of Smith [20] and reduces the conical Radon transform to the standard Radon transform. This further allows us to show stability of inverting the conical Radon transform. Generalizations of our results to higher dimension are presented in the appendix. 


\section{V-line transform with vertices on a circle}

In this section we study the the V-line transform on the circle. We derive an explicit inversion formula using a Fourier series expansion, which in particular implies the invertibility of the V-line transform, and further derive a numerical reconstruction algorithm based on our inversion formula.

\subsection{Explicit inversion formula}

For $\varphi \in[0,2 \pi)$ we write $\theta(\varphi):=(\cos \varphi, \sin \varphi)$. Further, we denote by $D_{1}(0):=\left\{x \in \mathbb{R}^{2} \mid\right.$ $\|x\|<1\}$ the unit disc in $\mathbb{R}^{2}$ and by $C_{c}^{\infty}\left(D_{1}(0)\right)$ the set of all $C^{\infty}$-functions $F: \mathbb{R}^{2} \rightarrow \mathbb{R}$ with $\operatorname{supp}(F) \subseteq D_{1}(0)$.

Definition 2.1 (The V-line transform on the circle). Let $F \in C_{c}^{\infty}\left(D_{1}(0)\right)$. We define the V-line transform of $F$ by

$$
\mathcal{V} F:[0,2 \pi) \times(0, \pi / 2) \rightarrow \mathbb{R}:(\varphi, \psi) \mapsto \sum_{\sigma= \pm 1} \int_{0}^{\infty} F(\theta(\varphi)-r \theta(\varphi-\sigma \psi)) \mathrm{d} r .
$$

The V-line transform integrates the function $F$ over V-lines (one-sided cones in the plane), having the vertex $\theta(\varphi):=(\cos \varphi, \sin \varphi) \in \mathbb{S}^{1}$, symmetry axis $\{-r \theta(\varphi) \mid r>0\}$ and half opening angle $\psi$. In the following we will frequently make use of the 2-dimensional (regular) Radon transform of a function $F \in C_{c}^{\infty}\left(\mathbb{R}^{2}\right)$, defined by

$$
(\mathcal{R} F)(\alpha, s):=\int_{\mathbb{R}} F(s \cos (\alpha)-t \sin (\alpha), s \sin (\alpha)+t \cos (\alpha)) \mathrm{d} t \quad \text { for }(\alpha, s) \in[0,2 \pi) \times \mathbb{R} .
$$

The Radon transform integrates the function $F$ over the line $\left\{x \in \mathbb{R}^{2} \mid(\cos (\alpha), \sin (\alpha)) \cdot x=\right.$ $s\}$ having a normal vector $(\cos (\alpha), \sin (\alpha))$ and an oriented distance $s \in \mathbb{R}$ from the origin.

The inversion approach we present below uses the Fourier series of $F$ and $\mathcal{V} F$ with respect the angular variables,

$$
\begin{aligned}
F(r \theta(\varphi)) & =\sum_{n \in \mathbb{Z}} F_{n}(r) e^{i n \varphi} \quad \text { with } \quad F_{n}(r):=\frac{1}{2 \pi} \int_{0}^{2 \pi} F(r \theta(\varphi)) e^{-i n \varphi} \mathrm{d} \varphi \\
(\mathcal{V} F)(\varphi, \psi) & =\sum_{n \in \mathbb{Z}}(\mathcal{V} F)_{n}(\psi) e^{i n \varphi} \quad \text { with } \quad(\mathcal{V} F)_{n}(\psi):=\frac{1}{2 \pi} \int_{0}^{2 \pi}(\mathcal{V} F)(\varphi, \psi) e^{-i n \varphi} \mathrm{d} \varphi .
\end{aligned}
$$

For $k \geq 0$, we denote by $T_{k}(z)$ and $U_{k}(z)$ the Chebyshev polynomials of the first and second kind, respectively, where

$$
\begin{aligned}
T_{k}(z) & := \begin{cases}\cos (k \arccos (z)) & \text { for }|z| \leq 1 \\
\cosh (k \operatorname{arccosh}(z)) & \text { for } z>1 \\
(-1)^{k} \cosh (k \operatorname{arccosh}(-z)) & \text { for } z<-1\end{cases} \\
U_{k}(z): & = \begin{cases}\sin ((k+1) \arccos (z)) / \sin (\arccos (z)) & \text { for }|z| \leq 1 \\
\sinh ((k+1) \operatorname{arccosh}(z)) / \sinh (\operatorname{arccosh}(z)) & \text { for } z>1 \\
(-1)^{k} \sinh ((k+1) \operatorname{arccosh}(-z)) / \sinh (\operatorname{arccosh}(-z)) & \text { for } z<-1,\end{cases}
\end{aligned}
$$


and set $U_{-1}:=0$.

Our strategy for inverting $\mathcal{V}$ is to recover the Fourier coefficient $F_{n}$ from $(\mathcal{V} F)_{n}$ for any $n \in \mathbb{Z}$. For that purpose we proceed by setting up a one-dimensional integral equation for $F_{n}$ in terms of $(\mathcal{R} F)_{n}$ that will subsequently be solved explicitly.

Lemma 2.2 (Expressing $(\mathcal{V} F)_{n}$ in terms of $\left.F_{n}\right)$. Suppose $F \in C_{c}^{\infty}\left(D_{1}(0)\right)$ and let $F_{n}$ and $(\mathcal{V} F)_{n}$ denote the Fourier coefficients of $F$ and $\mathcal{V} F$ as defined in (2.2) and (2.3), respectively. Then, for all $(n, \psi) \in \mathbb{Z} \times(0, \pi / 2)$, we have

$$
(\mathcal{V} F)_{n}(\psi)=4 \cos (n(\psi-\pi / 2)) \int_{\sin (\psi)}^{1} F_{n}(r) \frac{T_{|n|}(\sin (\psi) / r)}{\sqrt{r^{2}-\sin ^{2}(\psi)}} \mathrm{d} r .
$$

Proof. From the definitions of $\mathcal{V} F$ and $\mathcal{R} F$ we have the following relation

$$
\mathcal{V} F(\varphi, \psi)=\mathcal{R} F(\varphi-\psi+\pi / 2, \sin (\psi))+\mathcal{R} F(\varphi+\psi-\pi / 2, \sin (\psi)) .
$$

Now let $(\mathcal{R} F)_{n}(s):=\frac{1}{2 \pi} \int_{0}^{2 \pi}(\mathcal{R} F)(\alpha, s) e^{-i n \alpha} \mathrm{d} \alpha$ denote the $n$-th Fourier coefficient of $\mathcal{R} F$ with respect to the angular variable. Equation (2.5), the definition of the Fourier coefficients of $\mathcal{V} F$ and $\mathcal{R} F$, and two variable substitutions yield

$$
\begin{aligned}
& (\mathcal{V} F)_{n}(\psi) \\
& =\frac{1}{2 \pi} \int_{0}^{2 \pi}[\mathcal{R} F(\varphi-\psi+\pi / 2, \sin (\psi))+\mathcal{R} F(\varphi+\psi-\pi / 2, \sin (\psi))] e^{-i n \varphi} \mathrm{d} \varphi \\
& =\frac{1}{2 \pi} \int_{0}^{2 \pi} \mathcal{R} F(\alpha, \sin (\psi)) e^{-i n(\alpha+\psi-\pi / 2)} \mathrm{d} \alpha+\frac{1}{2 \pi} \int_{0}^{2 \pi} \mathcal{R} F(\alpha, \sin (\psi)) e^{-i n(\alpha-\psi+\pi / 2)} \mathrm{d} \alpha \\
& =(\mathcal{R} F)_{n}(\sin (\psi)) e^{-i n(\psi-\pi / 2)}+(\mathcal{R} F)_{n}(\sin (\psi)) e^{i n(\psi-\pi / 2)} \\
& =2 \cos (n(\psi-\pi / 2))(\mathcal{R} F)_{n}(\sin (\psi)) .
\end{aligned}
$$

Next we note the relation $(\mathcal{R} F)_{n}(s)=2 \int_{s}^{1} F_{n}(r) \frac{T_{|n|}(s / r)}{\sqrt{r^{2}-s^{2}}} \mathrm{~d} r$, that was first derived by Cormack in [4]. Combining this with the last displayed equation yields (2.4).

Lemma 2.2 together with known inversion formulas for the Radon transform yields the following explicit inversion formulas for the $\mathrm{V}$-line transform on the circle.

Theorem 2.3 (Inversion of the V-line transform on the circle). Suppose $F \in C^{\infty}\left(D_{1}(0)\right.$ ) and let $F_{n}$ and $(\mathcal{V} F)_{n}$ denote the Fourier coefficients of $F$ and $\mathcal{V} F$, as defined in (2.2) and (2.3). Then the following inversion formulas hold:

$$
\begin{aligned}
F_{n}(r)=-\frac{1}{2 \pi} \int_{r}^{1} \frac{\partial}{\partial s}\left[\frac{(\mathcal{V} F)_{n}(\arcsin (s))}{\cos (n(\arcsin (s)-\pi / 2))}\right] \frac{T_{|n|}(s / r)}{\sqrt{s^{2}-r^{2}}} \mathrm{~d} s \\
\begin{aligned}
F_{n}(r)=-\frac{1}{2 \pi r}\left\{\int_{r}^{1} \frac{\partial}{\partial s}\right. & {\left[\frac{(\mathcal{V} F)_{n}(\arcsin (s))}{\cos (n(\arcsin (s)-\pi / 2))}\right] \frac{\left[s / r+\sqrt{s^{2} / r^{2}-1}\right]^{-|n|}}{\sqrt{s^{2} / r^{2}-1}} \mathrm{~d} s } \\
& \left.-\int_{0}^{r} \frac{\partial}{\partial s}\left[\frac{(\mathcal{V} F)_{n}(\arcsin (s))}{\cos (n(\arcsin (s)-\pi / 2))}\right] U_{|n|-1}(s / r) \mathrm{d} s\right\} .
\end{aligned}
\end{aligned}
$$


Proof. Let $(\mathcal{R} F)_{n}$ denote the $n$-th Fourier coefficient of $\mathcal{R} F$. Then (2.6) implies

$$
(\mathcal{R} F)_{n}(s)=\frac{(\mathcal{V} F)_{n}(\arcsin (s))}{2 \cos (n(\arcsin (s)-\pi / 2))} .
$$

Next we recall the following inversion formulas for the Radon transform:

$$
\begin{aligned}
& F_{n}(r)=-\frac{1}{\pi} \int_{r}^{1} \frac{\partial(\mathcal{R} F)_{n}(s)}{\partial s} \frac{T_{|n|}(s / r)}{\sqrt{s^{2}-r^{2}}} \mathrm{~d} s \\
& F_{n}(r)=-\frac{1}{\pi r}\left\{\int_{r}^{1} \frac{\partial(\mathcal{R} F)_{n}(s)}{\partial s} \frac{\left[s / r+\sqrt{s^{2} / r^{2}-1}\right]^{-|n|}}{\sqrt{s^{2} / r^{2}-1}} \mathrm{~d} s\right. \\
&\left.\quad-\int_{0}^{r} \frac{\partial(\mathcal{R} F)_{n}(s)}{\partial s} U_{|n|-1}(s / r) \mathrm{d} s\right\} .
\end{aligned}
$$

Here (2.10) is Cormack's inversion formula [4] and (2.11) an inversion formula with better stability properties first derived in [17]. Inserting (2.9) in (2.10) yields the inversion formula (2.7) whereas inserting (2.11) yields (2.8).

Theorem 2.3 in particular implies that the V-line transform is uniquely invertible. Further, it implies the following inversion method for the V-line transform:

Algorithm 1 (Inversion of the V-line transform).

* Step 1: Compute the Fourier coefficients $(\mathcal{V} F)_{n}$ of $\mathcal{V} F$; see (2.3).

* STEP 2: Recover the Fourier coefficients $F_{n}$ from $(\mathcal{V} F)_{n}$ by (2.7) or (2.8)).

* STEP 3: Recover F from its Fourier coefficients $F_{n}$; see (2.2).

The inversion formula (2.7) solves the exterior problem for the V-line transform, because for reconstructing $F_{n}(r)$ it only uses integrals over $\mathrm{V}$-lines that do not intersect the disc $\left\{x \in \mathbb{R}^{2}|| x \mid \leq r\right\}$. Such data can be stably obtained from exterior data of the Radon transform. As a consequence, evaluating (2.7) is numerically unstable (severely ill-posed). For the following, we therefore only consider the inversion formula (2.8) and we demonstrate that it can be evaluated stably and efficiently.

\section{$2.2 \quad$ Numerical implementation}

In this subsection we describe how to numerically implement Algorithm 1. In our implementation, we discretize any step in Algorithm 1. For computing the Fourier coefficients in Step 1 and for evaluating the Fourier series in Step 3, we use the standard FFT algorithm. For Step 1, the FFT algorithm outputs approximations $\mathrm{G}[n, j] \simeq(\mathcal{V} F)_{n}\left(\arcsin \left(s_{j}\right)\right)$ for $n \in\{-N / 2,-N / 2+1, \ldots, N / 2-1\}$ and $j \in\{0, \ldots, M\}$. 
The main issue in the reconstruction procedure is implementing the inversion formula (2.8) in Step 2. Consider equidistant grid points $r_{i}=i / M$ for $i \in\{0, \ldots, M\}$ and rewrite the inversion formula (2.8) in the form

$$
\begin{aligned}
& F_{n}(r)=\frac{1}{\pi}\left\{\int_{0}^{r} g_{n}^{\prime}(s) U_{|n|-1}(s / r) \frac{\mathrm{d} s}{r}-\int_{r}^{1} g_{n}^{\prime}(s) \frac{\left[s / r+\sqrt{s^{2} / r^{2}-1}\right]^{-|n|}}{\sqrt{s^{2} / r^{2}-1}} \frac{\mathrm{d} s}{r}\right\}, \\
& g_{n}^{\prime}(s):=\frac{\partial}{\partial s}\left[\frac{(\mathcal{V} F)_{n}(\arcsin (s))}{2 \cos (n(\arcsin (s)-\pi / 2))}\right] .
\end{aligned}
$$

These formulas are used for finding approximations to $F_{n}\left(r_{i}\right)$ as follows. First, for some small regularization parameter $\epsilon>0$ (that accounts for instabilities due to the zeros in the denominator in the definition to $g_{n}^{\prime}$ ) we define

$$
\mathrm{H}[n, j]:=\frac{\cos \left(n\left(\arcsin \left(s_{j}\right)-\pi / 2\right)\right) \mathrm{G}[n, j]}{\epsilon^{2}+\cos \left(n\left(\arcsin \left(s_{j}\right)-\pi / 2\right)\right)^{2}},
$$

and take $g_{n}^{\prime}[j]:=(\mathrm{H}[n, j+1]-\mathrm{H}[n, j]) /(2 M)$ as an approximation of $g_{n}^{\prime}(s)$ on the interval $\left[r_{j}, r_{j+1}\right]$. Using such an approximation, we obtain

$$
\begin{aligned}
& F_{n}\left(r_{i}\right)= \frac{1}{\pi}\left\{\int_{0}^{r_{i}} g_{n}^{\prime}(s) U_{|n|-1}\left(s / r_{i}\right) \frac{\mathrm{d} s}{r_{i}}-\int_{r_{i}}^{1} g_{n}^{\prime}(s) \frac{\left[s / r_{i}+\sqrt{s^{2} / r_{i}^{2}-1}\right]^{-|n|}}{\sqrt{s^{2} / r_{i}^{2}-1}} \frac{\mathrm{d} s}{r_{i}}\right\} \\
&= \frac{1}{\pi}\left\{\sum_{j=0}^{i-1} \int_{r_{j}}^{r_{j+1}} g_{n}^{\prime}(s) U_{|n|-1}\left(s / r_{i}\right) \frac{\mathrm{d} s}{r_{i}}\right. \\
&\left.-\sum_{j=i}^{M-1} \int_{r_{j}}^{r_{j+1}} g_{n}^{\prime}(s) \frac{\left[s / r_{i}+\sqrt{s^{2} / r_{i}^{2}-1}\right]^{-|n|}}{\sqrt{s^{2} / r_{i}^{2}-1}} \frac{\mathrm{d} s}{r_{i}}\right\} \\
& \simeq \frac{1}{\pi}\left\{\sum_{j=0}^{i-1} g_{n}^{\prime}[j] \int_{r_{j}}^{r_{j+1}} U_{|n|-1}\left(s / r_{i}\right) \frac{\mathrm{d} s}{r_{i}}\right. \\
&\left.-\sum_{j=i}^{N-1} g_{n}^{\prime}[j] \int_{r_{j}}^{r_{j+1}} \frac{\left[s / r_{i}+\sqrt{s^{2} / r_{i}^{2}-1}\right]^{-|n|}}{\sqrt{s^{2} / r_{i}^{2}-1}} \frac{\mathrm{d} s}{r_{i}}\right\} .
\end{aligned}
$$

By elementary integration one verifies that

$$
\begin{array}{rlrl}
\forall j \in\{0, \ldots, i-1\}: w_{i, j}^{(n)} & :=\int_{r_{j}}^{r_{j+1}} U_{|n|-1}\left(s / r_{i}\right) \frac{\mathrm{d} s}{r_{i}} & \\
& = \begin{cases}0 & \text { for } n=0 \\
\frac{1}{|n|}\left(T_{|n|}\left(r_{j+1} / r_{i}\right)-T_{|n|}\left(r_{j} / r_{i}\right)\right) & \text { for } n \neq 0\end{cases}
\end{array}
$$

and

$$
\forall j \in\{i, \ldots, M-1\}: \quad w_{i, j}^{(n)}:=\int_{r_{j}}^{r_{j+1}} \frac{\left[s / r_{i}+\sqrt{s^{2} / r_{i}^{2}-1}\right]^{-|n|}}{\sqrt{s^{2} / r_{i}^{2}-1}} \frac{\mathrm{d} s}{r_{i}}
$$




$$
= \begin{cases}\log \left(r_{j+1}+\sqrt{r_{j+1}^{2}-r_{i}^{2}}\right)-\log \left(r_{j}+\sqrt{r_{j}^{2}-r_{i}^{2}}\right) & \text { for } n=0 \\ -\frac{1}{|n|}\left(e^{-|n| \operatorname{arccosh}\left(r_{j+1} / r_{i}\right)}-e^{-|n| \operatorname{arccosh}\left(r_{j} / r_{i}\right)}\right) & \text { for } n \neq 0 .\end{cases}
$$

Consequently, we obtain

$$
\forall(n, i) \in\{-N / 2, \ldots, N / 2-1\} \times\{1, \ldots, M\}: \quad \mathrm{F}[n, i]=\sum_{j=0}^{M-1} w_{i, j}^{(n)} \frac{\mathrm{H}[n, j]-\mathrm{H}[n, j]}{2 M}
$$

where $\mathrm{H}[n, j]$ is given by (2.12) , the weights $w_{i, j}^{(n)}$ are defined by (2.13) and (2.14) , and $\mathrm{F}[n, i]$ is the desired approximation to $F_{n}\left(r_{i}\right)$.

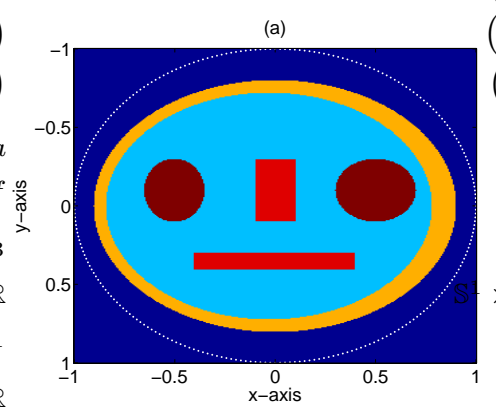

Figure 2.1: (a) Phantom $F$ used for numerical simulations. transform $\mathcal{V} F$. (c) Corresponding X-ray transform $\mathcal{X} F$.

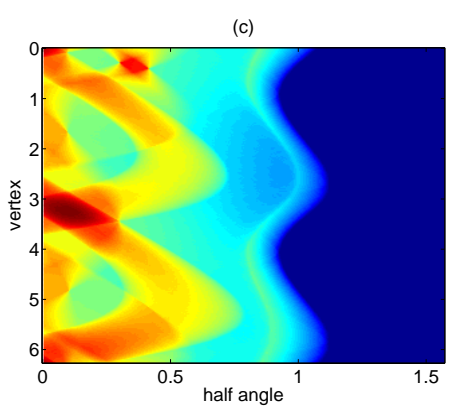

(b) Corresponding V-line

Formula (2.15) immediately yields to the following discrete reconstruction algorithm for inverting the $\mathrm{V}$-line transform using discrete data.

Algorithm 2 (Discrete reconstruction algorithm for inverting the V-line transform).

* STEP 1: Use the FFT to compute the $\mathrm{G}[n, i] \simeq(\mathcal{V} F)_{n}\left(s_{i}\right)$.

* STEP 2: For some $\epsilon>0$, compute $\mathrm{H}[n, i] \simeq(\mathcal{R} F)_{n}\left(s_{i}\right)$ by evaluating (2.12).

* STEP 3: Compute $\mathrm{F}[n, i] \simeq \mathrm{F}_{n}\left(r_{i}\right)$ by evaluating (2.15).

* STEP 4: Approximate $\mathrm{F}\left(r_{i} \cos \varphi_{k}, r_{i} \cos \varphi_{k}\right)$ by applying the inverse FFT.

Algorithm 2 is numerically efficient in the following sense. If $M=\mathcal{O}(N)$, easy arguments show that the proposed algorithm only requires $\mathcal{O}\left(N^{3}\right)$ floating point operations for reconstructing the phantom at $N^{2}$ reconstruction points. This is the same complexity as filtered backprojection reconstruction algorithms have. Notice further that by evaluating (2.15) in Step 3 in Algorithm 2 we actually implement the inversion formula (2.11) for the regular Radon transform derived by Perry [17]. The proposed implementation of (2.11) is of interest on its own and is different from the implementation given in [10]. Finally note that a 
different reconstruction strategy based on (2.12) (or (2.9)) would be to first recover $\mathcal{R} F$ by applying the inverse FFT algorithm and then to apply any existing reconstruction algorithm for the Radon transform such as the filtered backprojection algorithm.

\subsection{Numerical examples}

The reconstruction procedure outlined above has been implemented in MATLAB and tested on a discretized version of a Smiley phantom shown in Figure 2.1 (a) sampled on a Cartesian $201 \times 201$ grid. For implementing the V-line transform we first numerically computed the X-ray transform $\mathcal{X} F(\varphi, \psi)=\int_{0}^{\infty} F(\theta(\varphi)-r(\cos (\varphi-\psi), \sin (\varphi-\psi))) \mathrm{d} r$ by computing the ray integrals using the composite trapezoidal rule. We then evaluated the $\mathrm{V}$-line transform using $\mathcal{V} F(\varphi, \psi)=\sum_{\sigma= \pm 1} \mathcal{X} F(\varphi, \sigma \psi)$. Figure 2.1(b) and (c) show the numerically computed $\mathrm{V}$-line and X-ray transforms for $m=256$ vertex positions and $N=201$ opening angles in the interval $[0, \pi / 2]$.

(a)

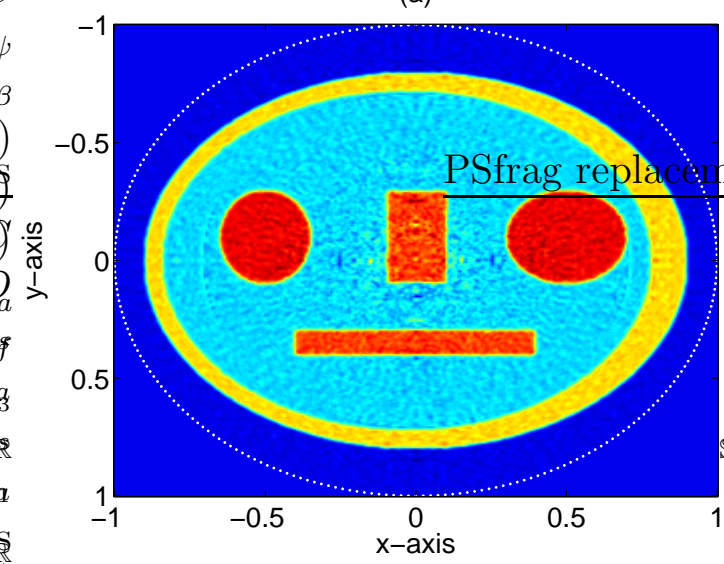

(c)

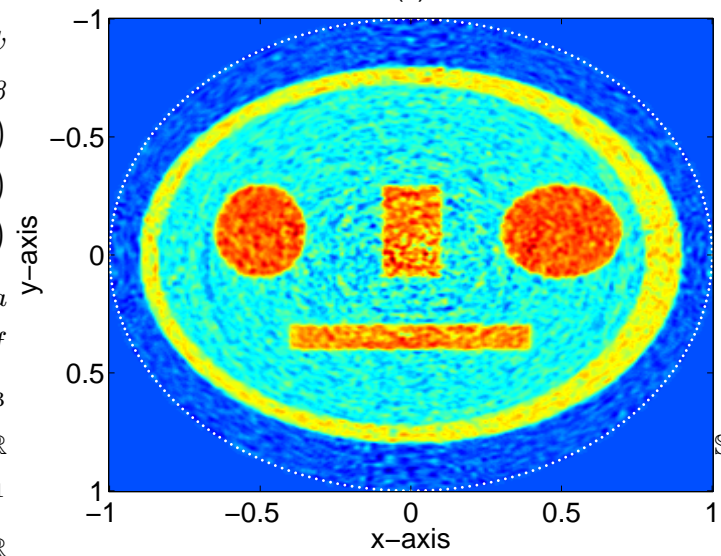

(b)

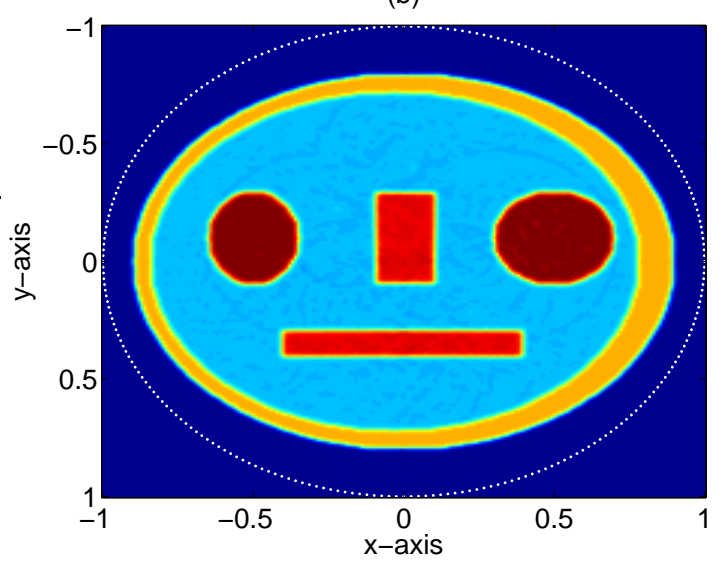

(d)

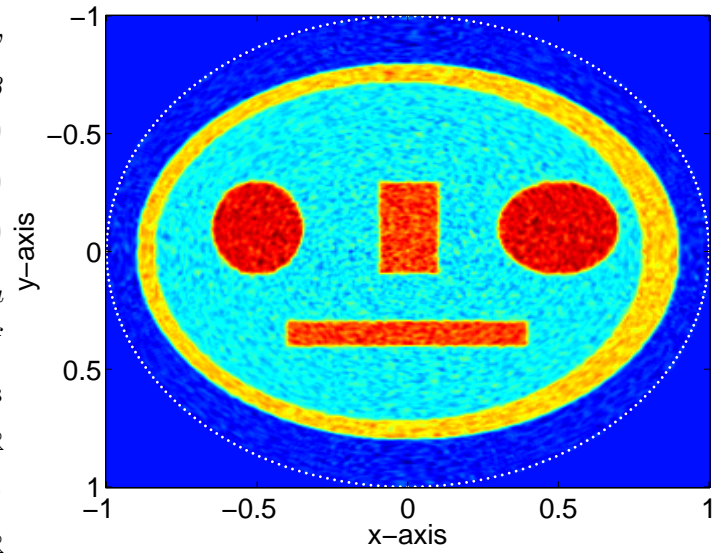

Figure 2.2: (a) Reconstruction from numerically computed V-line transform. (b) Reconstruction from numerically computed X-ray transform. (c) Reconstruction from V-line transform after adding Gaussian white noise to the data. (d) Reconstruction from X-ray transform after adding Gaussian white noise to the data. 
The numerical reconstruction from the V-line transform using Algorithm 2 evaluated on a Cartesian $201 \times 201$ grid is shown in Figure 2.2 (a). The regularization parameter has been taken as $\epsilon=0.005$. For comparison purpose we also applied our reconstruction algorithm to the X-ray transform, see Figure 2.2 (b). For inverting the X-ray transform, equation (2.12) is replaced by $\mathrm{H}[n, j]:=\mathrm{G}[n, j] / \exp \left(-i n\left(\arcsin \left(s_{j}\right)-\pi / 2\right)\right)$. (In fact, such a reconstruction procedure is justified by (2.5) and (2.6) $)$.) Because $\exp (-i n t) \neq 0$, no regularization is necessary for inverting $\mathcal{X} F$. Finally, in order to demonstrate the stability of our algorithm we added 5\% noise to the data and repeated the computations. For inverting the V-line transform we used an increased regularization parameter equal to $\epsilon=0.05$. Again the results for both the $\mathrm{V}$-line and the X-ray transform are quite good. However, the inversion of the $\mathrm{V}$-line transform is slightly more sensitive to the noise which is expected due to the zeros of the function $s \mapsto \cos (n(\arcsin (s)-\pi / 2))$ appearing in the denominator of the inversion formula for $\mathcal{V} F$.

\section{The conical Radon transform with vertices on a cylin- der}

In this section we study the inversion of the conical Radon transform on the cylinder using $4 \mathrm{D}$ data. For that purpose, for $(\varphi, z, \beta, \psi) \in[0,2 \pi) \times \mathbb{R} \times(0, \pi)^{2}$, we use the following notations:

$$
\begin{aligned}
& * \theta(\varphi):=(\cos \varphi, \sin \varphi) \in \mathbb{S}^{1} ; \\
& * a(\varphi, \beta):=(-\theta(\varphi) \sin (\beta), \cos (\beta)) \in \mathbb{S}^{2} ; \\
& * C(\varphi, z, \beta, \psi):=(\theta(\varphi), z)+\left\{x \in \mathbb{R}^{3} \mid a(\varphi, \beta) \cdot x=\|x\| \cos \psi\right\} .
\end{aligned}
$$

The set $C(\varphi, z, \beta, \psi)$ is a one-sided circular cone, having a vertex $(\theta(\varphi), z) \in \mathbb{S}^{1} \times \mathbb{R}$, a symmetry axis $\{r a(\varphi, \beta) \mid r>0\}$ pointing to the symmetry axis of the cylinder $\mathbb{S}^{1} \times \mathbb{R}$, and a half opening angle $\psi \in(0, \pi)$; see Figure 1.2 (a).

Definition 3.1 (Conical Radon transform on the cylinder). Let $k \in \mathbb{N}_{0}=\{0,1, \ldots\}$ and $f \in C_{c}^{\infty}\left(D_{1}(0) \times \mathbb{R}\right)$. We define the (weighted) conical Radon transform of $f$ by

$$
\begin{aligned}
\mathcal{C}_{k} f:[0,2 \pi) \times \mathbb{R} \times(0, \pi)^{2} & \rightarrow \mathbb{R}: \\
(\varphi, z, \beta, \psi) & \mapsto \int_{C(\varphi, z, \beta, \psi)} f(x)\|x-(\theta(\varphi), z)\|^{k-1} \mathrm{~d} S(x) .
\end{aligned}
$$

The weighted conical Radon transform maps the function $f$ to integrals over members of the four-dimensional set of all cones $\left\{C(\varphi, z, \beta, \psi) \subseteq \mathbb{R}^{3} \mid(\varphi, z, \beta, \psi) \in[0,2 \pi) \times \mathbb{R} \times(0, \pi)^{2}\right\}$. The parameter $k \in \mathbb{N}_{0}$ allows to include a radial weight that can be adjusted to a particular application at hand. In the literature on Compton camera imaging the case $k=0$ and $k=1$ have been used. In [20], the case $k=1$ is referred to as the surface integral model, and the case $k=0$ as the cone-beam line integral model.

In the following we present two inversion methods for inverting $\mathcal{C}_{k}$. The first one is based on reducing the conical Radon transform to the V-line transform, whereas the second one is based on reducing the conical Radon transform to the Radon transform. 


\subsection{Method 1: Reduction to the V-line transform}

The first inversion method consists in first recovering the $\mathrm{V}$-line transform from $\mathcal{C}_{k} f$ and subsequently recovering $f$ by inverting the $\mathrm{V}$-line transform. For that purpose we make use of the (weighted) X-ray transform

$$
\left.\left(\mathcal{X}_{k} f\right):[0,2 \pi) \times \mathbb{R} \times \mathbb{R}^{3} \rightarrow \mathbb{R}:(\varphi, z, u) \mapsto \int_{0}^{\infty} f((\theta(\varphi), z)+r u)\right) r^{k} \mathrm{~d} r
$$

for $k \in \mathbb{N}_{0}$ and $f \in C_{c}^{\infty}\left(D_{1}(0) \times \mathbb{R}\right)$. The X-ray transform consists of integrals over rays $\{(\theta(\varphi), z)+r u \mid r>0\}$ with a vertex $(\theta(\varphi), z) \in \mathbb{S}^{1} \times \mathbb{R}$ and a direction $u \in \mathbb{R}^{3}$.

Lemma 3.2. Let $f \in C_{0}^{\infty}\left(D_{1}(0) \times \mathbb{R}\right)$ and $(\varphi, z) \in[0,2 \pi) \times \mathbb{R}$. For any $y=\left(y_{1}, y_{2}\right) \in \mathbb{R}^{2}$ with $\|y\| \leq 1$, we have

$$
\begin{aligned}
\sum_{\sigma= \pm 1}\left(\mathcal{X}_{k} f\right)\left(\varphi, z, y_{1} \theta(\varphi)\right. & \left.+\sigma \sqrt{1-\|y\|^{2}} \theta(\varphi)^{\perp}, y_{2}\right) \\
& =\frac{\sqrt{1-\|y\|^{2}}}{2 \pi^{2}} \int_{0}^{\pi} \int_{0}^{\pi} \frac{\partial_{\psi}\left[\mathcal{C}_{k} f(\varphi, z, \alpha, \psi) / \sin \psi\right]}{\cos (\psi)-y \bullet(-\sin (\beta), \cos (\beta))} \mathrm{d} \psi \mathrm{d} \beta
\end{aligned}
$$

Further, for any $v \in \mathbb{S}^{1}$ we have

$$
\left(\mathcal{X}_{0} f\right)(\varphi, z, v, 0)=\frac{(-1)^{k-1}}{(k-1) !} \int_{-\pi / 2}^{0} \sin ^{k-1}(\gamma)\left(\partial_{z}^{k} \mathcal{X}_{k} f\right)(\varphi, z, v \cos (\gamma), \sin (\gamma)) \mathrm{d} \gamma
$$

Proof. Using the one-dimensional delta-distribution, we have

$$
\begin{aligned}
\mathcal{C}_{k} f(\varphi, z, \beta, \psi) & =\sin (\psi) \int_{\mathbb{S}^{2}} \int_{0}^{\infty} f((\varphi, z)+r \omega) \delta(a(\varphi, \beta) \cdot \omega-\cos (\psi)) r^{k} \mathrm{~d} r \mathrm{~d} S(\omega) \\
& =\sin (\psi) \int_{\mathbb{S}^{2}}\left(\mathcal{X}_{k} f\right)(\varphi, z, \omega) \delta(a(\varphi, \beta) \cdot \omega-\cos (\psi)) \mathrm{d} S(\omega) \\
& =\sum_{\sigma= \pm 1} \sin (\psi) \int_{\mathbb{S}_{\sigma}^{2}}\left(\mathcal{X}_{k} f\right)(\varphi, z, \omega) \delta(a(\varphi, \beta) \cdot \omega-\cos (\psi)) \mathrm{d} S(\omega)
\end{aligned}
$$

where $\mathbb{S}_{\sigma}^{2}:=\left\{\omega \in \mathbb{S}^{2} \mid\left(\sigma \theta(\varphi)^{\perp}, 0\right) \bullet \omega>0\right\}$. Any element on the half sphere $\mathbb{S}_{\sigma}^{2}$ can uniquely be written in the form $\omega=\left(y_{1} \theta(\varphi)+\sigma \sqrt{1-\|y\|^{2}} \theta(\varphi)^{\perp}, y_{2}\right)$ for $y=\left(y_{1}, y_{2}\right) \in \mathbb{R}^{2}$ with $\|y\|<1$. Using this representation we have $a(\varphi, \beta) \bullet \omega=(-\sin (\beta), \cos (\beta)) \cdot y$. Together with (3.5) and the transformation rule this shows

$$
\begin{aligned}
\mathcal{C}_{k} f(\varphi, z, \beta, \psi)=\sum_{\sigma= \pm 1} \int_{\|y\|<1}\left(\mathcal{X}_{k} f\right)(\varphi, z & \left.y_{1} \theta(\varphi)+\sigma \sqrt{1-\|y\|^{2}} \theta(\varphi)^{\perp}, y_{2}\right) \\
& \times \delta((-\sin (\beta), \cos (\beta)) \cdot y-\cos (\psi)) \frac{\sin (\psi) \mathrm{d} y}{\sqrt{1-\|y\|^{2}}} .
\end{aligned}
$$


Now for fixed $\varphi, z$ and $k$, we define functions $F_{\sigma}: \mathbb{R}^{2} \rightarrow \mathbb{R}$ by

$$
F_{\sigma}(y):= \begin{cases}\left(\mathcal{X}_{k} f\right)\left(\varphi, z, y_{1} \theta(\varphi)+\sqrt{1-\|y\|^{2}} \theta(\varphi)^{\perp}, y_{2}\right) \frac{1}{\sqrt{1-\|y\|^{2}}} & \text { for }\|y\| \leq 1 \\ 0 & \text { for }\|y\|>1\end{cases}
$$

and recall that the 2-dimensional Radon transform can be written in the form $\mathcal{R} F(\alpha, s)=$ $\int_{\mathbb{R}^{2}} F(y) \delta((\cos (\alpha), \sin (\alpha)) \cdot y-s) \mathrm{d} y$; we obtain $\mathcal{C}_{k} f(\varphi, z, \beta, \psi) / \sin \psi=\sum_{\sigma= \pm 1}\left(\mathcal{R} F_{\sigma}\right)(\beta+$ $\pi / 2, \cos (\psi))$. With the formula $F(y)=\frac{1}{2 \pi^{2}} \int_{0}^{\pi} \int_{\mathbb{R}}((\cos (\alpha), \sin (\alpha)) \cdot y-s)^{-1}\left(\partial_{s} \mathcal{R} F\right)(\alpha, s) \mathrm{d} s \mathrm{~d} \alpha$ for inverting the Radon transform (see, for example, [11, 16]) this yields

$$
\begin{gathered}
\sum_{\sigma= \pm 1} F_{\sigma}(y)=\frac{1}{2 \pi^{2}} \int_{0}^{\pi} \int_{-1}^{1} \frac{\partial_{\cos (\psi)}\left[\mathcal{C}_{k} f(\varphi, z, \alpha-\pi / 2, \psi) / \sin \psi\right]}{y \cdot(\cos (\alpha), \sin (\alpha))-\cos (\psi)} \mathrm{d}(\cos \psi) \mathrm{d} \alpha \\
=\frac{1}{2 \pi^{2}} \int_{0}^{\pi} \int_{0}^{\pi} \frac{\partial_{\psi}\left[\mathcal{C}_{k} f(\varphi, z, \alpha-\pi / 2, \psi) / \sin \psi\right]}{\cos (\psi)-(\cos (\alpha), \sin (\alpha)) \cdot y} \mathrm{~d} \psi \mathrm{d} \alpha
\end{gathered}
$$

Inserting the definition of $F_{\sigma}$ yields (3.3).

By the chain rule we have

$$
\begin{array}{rl}
\left(\partial_{w}^{k} \mathcal{X}_{0} f\right)(\varphi, z, v, w)=\int_{0}^{\infty} \partial_{w}^{k} & f((\theta(\varphi), z)+r(v, w)) \mathrm{d} r \\
=\int_{0}^{\infty} \partial_{z}^{k} f((\theta(\varphi), z)+r(v, w)) r^{k} \mathrm{~d} r=\left(\partial_{z}^{k} \mathcal{X}_{k} f\right)(\varphi, z, v, w)
\end{array}
$$

Together with Cauchy's formula for repeated integration we obtain

$$
\begin{aligned}
\left(\mathcal{X}_{0} f\right)(\varphi, z, v, w) & =\frac{1}{(k-1) !} \int_{-\infty}^{w}(w-s)^{k-1}\left(\partial_{z}^{k} \mathcal{X}_{k} f\right)(\varphi, z, v, s) \mathrm{d} s \\
= & \frac{1}{(k-1) !} \int_{-\infty}^{w}(w-s)^{k-1}\left(\partial_{z}^{k} \mathcal{X}_{k} f\right)\left(\varphi, z, \frac{(v, s)}{\sqrt{\|v\|^{2}+s^{2}}}\right) \frac{\mathrm{d} s}{\left(\|v\|^{2}+s^{2}\right)^{(k+1) / 2}}
\end{aligned}
$$

where for the second equality we used the identity $\left(\mathcal{X}_{k} f\right)(\varphi, z, \lambda u)=\lambda^{-k-1}\left(\mathcal{X}_{k} f\right)(\varphi, z, u)$ that holds for every $\lambda>0$ and $u \in \mathbb{R}^{3}$.

Now we take $w=0$ and $\|v\|=1$ in the last displayed equation, and make the substitution $s=\tan (\gamma)$. Then $\|v\|^{2}+s^{2}=1 / \cos ^{2}(\gamma)$ and $\mathrm{d} s=\mathrm{d} \gamma / \cos ^{2}(\gamma)$. Consequently,

$$
\begin{aligned}
&\left(\mathcal{X}_{0} f\right)(\varphi, z, v, 0)=\frac{1}{(k-1) !} \int_{-\pi / 2}^{0}(-\tan (\gamma))^{k-1}\left(\partial_{z}^{k} \mathcal{X}_{k} f\right)(\varphi, z,v \cos (\gamma), \sin (\gamma)) \\
& \times \cos ^{k-1}(\gamma) \mathrm{d} \gamma \quad \text { for } v \in \mathbb{S}^{1}
\end{aligned}
$$

which after simple manipulation yields (3.4).

The following theorem shows how to explicitly reduce the weighted conical Radon transform to the (two-dimensional) V-line transform applied in horizontal planes. 
Theorem 3.3 (Reduction of the conical Radon transform to the V-line transform). Let $f \in C_{c}^{\infty}\left(D_{1}(0) \times \mathbb{R}\right)$. Then, we have for $(\varphi, z, \psi) \in[0,2 \pi) \times \mathbb{R} \times(0, \pi / 2)$,

$$
\begin{aligned}
\sum_{\sigma= \pm 1}\left(\mathcal{X}_{0} f\right)(\varphi, z,-\cos (\psi) \theta(\varphi)+ & \left.\sigma \sin (\psi) \theta(\varphi)^{\perp}, 0\right) \\
& =\int_{0}^{\pi} \int_{0}^{\pi} \partial_{z}^{k} \partial_{\psi}\left[\mathcal{C}_{k} f(\varphi, z, \beta, \psi) / \sin \psi\right] H_{k}(\beta, \psi) \mathrm{d} \psi \mathrm{d} \beta
\end{aligned}
$$

with

$$
H_{k}(\beta, \psi):=\frac{(-1)^{k-1}}{2 \pi^{2}(k-1) !} \int_{-\pi / 2}^{0} \frac{\sin ^{k-1}(\gamma) \cos (\gamma) \sin (\psi) \mathrm{d} \gamma}{\cos (\psi)-(\cos (\gamma) \cos (\psi), \sin (\gamma)) \cdot(\sin (\beta), \cos (\beta))}
$$

Proof. By Lemma 3.2 we have

$$
\begin{aligned}
& \sum_{\sigma= \pm 1}\left(\mathcal{X}_{0} f\right)\left(\varphi, z,-\cos (\psi) \theta(\varphi)+\sigma \sin (\psi) \theta(\varphi)^{\perp}, 0\right) \\
& =\frac{(-1)^{k-1}}{(k-1) !} \sum_{\sigma= \pm 1} \int_{-\pi / 2}^{0} \sin ^{k-1}(\gamma) \\
& \quad \times\left(\partial_{z}^{k} \mathcal{X}_{k} f\right)\left(\varphi, z,-\cos (\gamma) \cos (\psi) \theta(\varphi)+\sigma \cos (\gamma) \sin (\psi) \theta(\varphi)^{\perp}, \sin (\gamma)\right) \mathrm{d} \gamma \\
& =\frac{(-1)^{k-1}}{(k-1) !} \partial_{z}^{k} \int_{-\pi / 2}^{0} \sin ^{k-1}(\gamma) \\
& \quad \times \sum_{\sigma= \pm 1}\left(\mathcal{X}_{k} f\right)\left(\varphi, z,-\cos (\gamma) \cos (\psi) \theta(\varphi)+\sigma \cos (\gamma) \sin (\psi) \theta(\varphi)^{\perp}, \sin (\gamma)\right) \mathrm{d} \gamma \\
& =\frac{(-1)^{k-1}}{2 \pi^{2}(k-1) !} \int_{-\pi / 2}^{0} \int_{0}^{\pi} \int_{0}^{\pi} \frac{\sin ^{k-1}(\gamma) \cos (\gamma) \sin (\psi) \partial_{z}^{k} \partial_{\psi}\left[\mathcal{C}_{k} f(\varphi, z, \beta, \psi) / \sin \psi\right] \mathrm{d} \psi \mathrm{d} \beta \mathrm{d} \gamma}{\cos (\psi)-(-\cos (\gamma) \cos (\psi), \sin (\gamma)) \cdot(-\sin (\beta), \cos (\beta))}
\end{aligned}
$$

After interchanging the order of integrations and using the definition of the kernel $H_{k}(\beta, \psi)$ yields the desired identity.

Let $f \in C_{c}^{\infty}\left(D_{1}(0) \times \mathbb{R}\right)$. For any given $z \in \mathbb{R}$ define $f_{z}:=f(\cdot, z)$. Therefore, the V-line transform of $f_{z}$ satisfies

$$
\begin{aligned}
\left(\mathcal{V} f_{z}\right)(\varphi, \psi) & =\sum_{\sigma= \pm 1} \int_{0}^{\infty} f(\theta(\varphi)-r(\cos (\varphi-\sigma \psi), \sin (\varphi-\sigma \psi)), z) \mathrm{d} r \\
& =\sum_{\sigma= \pm 1}\left(\mathcal{X}_{0} f\right)\left(\varphi, z,-\cos (\psi) \theta(\varphi)+\sigma \sin (\psi) \theta(\varphi)^{\perp}, 0\right) \\
& =\int_{0}^{\pi} \int_{0}^{\pi} \partial_{z}^{k} \partial_{\psi}\left[\mathcal{C}_{k} f(\varphi, z, \beta, \psi) / \sin \psi\right] H_{k}(\beta, \psi, \gamma) \mathrm{d} \psi \mathrm{d} \beta
\end{aligned}
$$

Here the second equality follows from the identity $-\cos (\psi) \theta(\varphi)+\sigma \sin (\psi) \theta(\varphi)^{\perp}=-(\cos (\varphi-$ $\sigma \psi), \sin (\varphi-\sigma \psi))$ and the last equality from Theorem 3.3. Equation (3.9) shows how to recover the $\mathrm{V}$-line transform $\mathcal{V} f_{z}$ from the conical Radon transform. By subsequently inverting 
the V-line transform one recovers $f$. In summary, we conclude the following inversion method for the conical Radon transform:

Algorithm 3 (Inversion of the conical Radon transform, Method 1).

- STEP 1: Compute the kernel $H_{k}$ defined in (3.7).

* STEP 2: Evaluate (3.9) to recover $\mathcal{V} f_{z}$ from $\mathcal{C}_{k} f$ for every $z \in \mathbb{R}$.

* STEP 3: For every $z \in \mathbb{R}$ recover $f_{z}$ from $\mathcal{V} f_{z}$ by means of Algorithm 1 .

Theorem 3.3 also implies that the considered conical Radon transform $f \mapsto \mathcal{C}_{k} f$ is uniquely invertible and can be reconstructed by Algorithm 3. How to efficiently implement Algorithm 3 is a topic of future work.

\subsection{Method 2: Reduction to the Radon Transform}

The second method is probably simpler than the method presented above, but only works for the cases $k=0,1$. It based on a relation between the conical Radon transform and the regular 3-dimensional Radon transform, that has first been first derived by Smith [20]. For that purpose we use the following additional notation:

* $(\mathcal{R} f)(\omega, s):=\int_{\omega^{\perp}} f(s \omega+y) \mathrm{d} S(y)$ for $(\omega, s) \in \mathbb{S}^{2} \times \mathbb{R}$ denotes the regular 3-dimensional Radon transform of $f \in C_{c}^{\infty}\left(\mathbb{R}^{3}\right)$;

* $(\mathcal{H} g)(\omega, s):=\frac{1}{\pi} \int_{\mathbb{R}} g(\omega, t) \mathrm{d} t /(s-t)$ for $(\omega, s) \in \mathbb{S}^{2} \times \mathbb{R}$ denotes the Hilbert transform in the second component of a function $g \in C_{c}^{\infty}\left(\mathbb{S}^{2} \times \mathbb{R}\right)$.

Lemma 3.4 (Reduction of the conical Radon transform to the Radon transform). Let $f \in$ $C_{c}^{\infty}\left(\mathbb{R}^{3}\right)$. Then, for $(\varphi, z, \beta) \in[0,2 \pi) \times \mathbb{R} \times(0, \pi)$, we have

$$
\begin{aligned}
\left(\mathcal{H} \partial_{s} \mathcal{R} f\right)(a(\varphi, \beta), z \cos (\beta)-\sin (\beta)) & =-\frac{1}{\pi} \int_{0}^{\pi}\left(\mathcal{C}_{0} f\right)(\varphi, z, \beta, \psi) \frac{\mathrm{d} \psi}{\cos (\psi)^{2}} \\
(\mathcal{H R} f)(a(\varphi, \beta), z \cos (\beta)-\sin (\beta)) & =-\frac{1}{\pi} \int_{0}^{\pi}\left(\mathcal{C}_{1} f\right)(\varphi, z, \beta, \psi) \frac{\mathrm{d} \psi}{\cos (\psi)} .
\end{aligned}
$$

Proof. Expressing $\mathcal{C}_{k}$ for $k=0,1$ in terms of the one-dimensional delta-distribution and performing several coordinate substitutions yield

$$
\int_{0}^{\pi} \mathcal{C}_{k} f(\varphi, z, \beta, \psi) \cos ^{k-2}(\psi) \mathrm{d} \psi
$$




$$
\begin{aligned}
& =\int_{0}^{\pi} \int_{\mathbb{S}^{2}} \int_{0}^{\infty} f((\theta(\varphi), z)+r \omega) \delta(a(\varphi, \beta) \cdot \omega-\cos (\psi)) r^{k} \mathrm{~d} r \mathrm{~d} S(\omega) \cos ^{k-2}(\psi) \mathrm{d}(\cos (\psi)) \\
& =\int_{\mathbb{S}^{2}} \int_{0}^{\infty} f((\theta(\varphi), z)+r \omega)(a(\varphi, \beta) \cdot \omega)^{k-2} r^{k} \mathrm{~d} r \mathrm{~d} S(\omega) \\
& =\int_{\mathbb{R}^{3}} f((\theta(\varphi), z)+x)(a(\varphi, \beta) \cdot x)^{k-2} \mathrm{~d} x \\
& =\int_{\mathbb{R}^{3}} f(x)(a(\varphi, \beta) \cdot x-a(\varphi, \beta) \cdot(\theta(\varphi), z))^{k-2} \mathrm{~d} x \\
& =\int_{\mathbb{R}} \int_{a(\varphi, \beta)^{\perp}} f(s a(\varphi, \beta)+y)(s+\sin (\beta)-z \cos (\beta))^{k-2} \mathrm{~d} y \mathrm{~d} s \\
& =\int_{\mathbb{R}}(\mathcal{R} f)(a(\varphi, \beta), s)(s+\sin (\beta)-z \cos (\beta))^{k-2} \mathrm{~d} s .
\end{aligned}
$$

Here the third equality follows after introducing spherical coordinates $x \leftarrow r \omega$, the fourth equality follows after the change of variables $x \leftarrow(\theta(\varphi), z)+x$, the fifth equality follows after the substitution $x \leftarrow s a(\varphi, \beta)+y$, and the last equality follows form the definition of the Radon transform. Now, the definition of the Hilbert transform and performing one integration by parts in the case $k=0$ yields (3.10), (3.11).

Using Lemma 3.4 we can recover $\mathcal{H} \partial_{s}^{1-k} \mathcal{R} f$ from the conical Radon transform. By applying the inverse Hilbert transform and the inverse Radon transform afterwards one then recovers the original function. This yields the following reconstruction method.

Algorithm 4 (Inversion of the conical Radon transform for $k=0,1$, Method 2).

* STEP 1: Recover $\mathcal{H} \partial_{s}^{1-k} \mathcal{R} f$ from $\mathcal{C}_{k} f$ by evaluating (3.10) or (3.11).

* STEP 2: Recover $\partial_{s}^{1-k} \mathcal{R} f$ from $\mathcal{H} \partial_{s}^{1-k} \mathcal{R} f$ by applying inverse Hilbert transform.

* STEP 3: Recover $f$ from $\partial_{s}^{1-k} \mathcal{R} f$ by applying the inverse Radon transform.

While Algorithm 4 can efficiently be implemented, one can combine three steps to obtain an explicit inversion formula. Such inversion formulas are also useful for theoretical investigations. By using the standard filtered backprojection type inversion formulas for the Radon transform (see, for example, [11, 16])

$$
\begin{aligned}
& f(x)=-\frac{1}{8 \pi^{2}} \Delta_{x} \int_{\mathbb{S}^{2}} \mathcal{R} f(\omega, \omega \cdot x) \mathrm{d} S(\omega) \\
& f(x)=-\frac{1}{8 \pi^{2}} \int_{\mathbb{S}^{2}} \partial_{t}^{2} \mathcal{R} f(\omega, \omega \cdot x) \mathrm{d} S(\omega),
\end{aligned}
$$

Lemma 3.4 yields the following result. 
Theorem 3.5. For $f \in C^{\infty}\left(D_{1}(0) \times \mathbb{R}\right)$ we have

$$
\begin{array}{ll}
f(x)=-\frac{1}{8 \pi^{4}} \int_{\mathbb{S}^{2}} \int_{\mathbb{R}} \int_{0}^{\pi} \frac{\left(\partial_{z}^{2} \mathcal{C}_{k} f\right)(\varphi, z, \beta, \psi) \cos ^{-k}(\beta) \mathrm{d} \psi \mathrm{d} z \mathrm{~d} S(a(\varphi, \beta))}{(x \cdot a(\varphi, \beta)-z \cos (\beta)+\sin (\beta)) \cos ^{2-k}(\psi)} & \text { for } k \in\{0,1\} \\
f(x)=-\frac{1}{8 \pi^{4}} \Delta_{x} \int_{\mathbb{S}^{2}} \int_{\mathbb{R}} \int_{0}^{\pi} \frac{\mathcal{C}_{1} f(\varphi, z, \beta, \psi) \cos (\beta) \mathrm{d} \psi \mathrm{d} z \mathrm{~d} S(a(\varphi, \beta))}{(x \cdot a(\varphi, \beta)-z \cos (\beta)+\sin (\beta)) \cos (\psi)} \quad \text { for } k=0 .
\end{array}
$$

Proof. Since $\mathcal{H} \mathcal{H} g=-g$, we have

$$
\begin{aligned}
\partial_{s}^{1-k} \mathcal{R} f(a(\varphi, \beta), s) & =-\partial_{s}^{1-k}(\mathcal{H} \mathcal{H} \mathcal{R} f)(a(\varphi, \beta), s) \\
& =\frac{(-1)^{k}}{\pi} \int_{\mathbb{R}} \frac{\mathcal{H} \mathcal{R} f(a(\varphi, \beta), z \cos (\beta)-\sin (\beta)) \cos (\beta) \mathrm{d} z}{(s-z \cos (\beta)+\sin (\beta))^{2-k}} \\
& =-\frac{1}{\pi} \int_{\mathbb{R}} \frac{\partial_{z}^{1-k} \mathcal{H} \mathcal{R} f(a(\varphi, \beta), z \cos (\beta)-\sin (\beta)) \cos ^{k}(\beta) \mathrm{d} z}{s-z \cos (\beta)+\sin (\beta)} \\
& =\frac{1}{\pi^{2}} \int_{\mathbb{R}} \int_{0}^{\pi} \frac{\partial_{z}^{1-k} \mathcal{C}_{k} f(\varphi, z, \beta, \psi) \cos (\beta) \mathrm{d} \psi \mathrm{d} z}{(s-z \cos (\beta)+\sin (\beta)) \cos ^{2-k}(\psi)} .
\end{aligned}
$$

Together with (3.12) this yields (3.15) for $k=1$. Further, integration by parts shows

$$
\begin{aligned}
& \left(\partial_{s}^{2} \mathcal{R} f\right)(a(\varphi, \beta), t) \\
& =\left(\partial_{s}^{1+k} \partial_{s}^{1-k} \mathcal{R} f\right)(a(\varphi, \beta), t) \\
& =\frac{(-1)^{k+1}(k+1) !}{\pi^{2}} \int_{\mathbb{R}} \int_{0}^{\pi} \frac{\left(\partial_{z}^{1-k} \mathcal{C}_{k} f\right)(\varphi, z, \beta, \psi) \cos (\beta) \mathrm{d} \psi \mathrm{d} z}{(s-z \cos (\beta)+\sin (\beta))^{2+k} \cos ^{2-k}(\psi)} \\
& =\frac{(-1)^{k+1}}{\pi^{2}} \int_{\mathbb{R}}^{\pi} \int_{0}^{\pi} \partial_{z}^{1+k}\left[\frac{1}{s-z \cos (\beta)+\sin (\beta)}\right]\left(\partial_{z}^{1-k} \mathcal{C}_{k} f\right)(\varphi, z, \beta, \psi) \cos ^{-k}(\beta) \frac{\mathrm{d} \psi \mathrm{d} z}{\cos ^{2-k}(\psi)} \\
& =\frac{1}{\pi^{2}} \int_{\mathbb{R}} \int_{0}^{\pi} \frac{\left(\partial_{z}^{2} \mathcal{C}_{k} f\right)(\varphi, z, \beta, \psi) \cos ^{-k}(\beta)}{(s-z \cos (\beta)+\sin (\beta)) \cos ^{2-k}(\psi)} \mathrm{d} \psi \mathrm{d} z,
\end{aligned}
$$

which together with (3.13) yields (3.14).

The inversion formulas (3.14) for $k=0$ and (3.15) for $k=1$ can further be rewritten in terms of the conical back-projection operator $\mathcal{C}_{k}^{\sharp} g$ that is defined by

$$
\begin{aligned}
& \mathcal{C}_{k}^{\sharp} g(x)=\int_{0}^{\pi} \int_{0}^{\pi} \int_{\mathbb{R}} \int_{0}^{2 \pi} g(\varphi, z, \beta, \psi)\|x-(\theta(\varphi), z)\|^{k-1} \\
& \quad \times \delta(a(\varphi, \beta) \cdot x-z \cos (\beta)+\sin (\beta)-\|x-(\theta(\varphi), z)\| \cos (\psi)) \sin (\psi) \mathrm{d} \varphi \mathrm{d} z \mathrm{~d} \beta \mathrm{d} \psi .
\end{aligned}
$$

for $g \in C^{\infty}\left([0,2 \pi) \times \mathbb{R} \times(0, \pi)^{2}\right)$ and $x \in \mathbb{R}^{3}$. As shown in Appendix A.1, the operator $\mathcal{C}_{k}^{\sharp}$ is the (formal) $L^{2}$-adjoint of $\mathcal{C}_{k}$.

Corollary 3.6. Let $f \in C^{\infty}\left(D_{1}(0) \times \mathbb{R}\right)$. Then we have for $x \in \mathbb{R}^{3}$,

$$
f(x)=\frac{(-1)^{k}}{8 \pi^{4}} \Delta_{x}^{k} \mathcal{C}_{k}^{\sharp}\left[\int_{0}^{\pi} \frac{\partial_{z}^{1-k} \mathcal{C}_{k} f\left(\varphi, z, \beta, \psi^{\prime}\right) \cos (\beta) \sin (\beta) \mathrm{d} \psi^{\prime}}{\cos ^{2-k}(\psi) \cos ^{2-k}\left(\psi^{\prime}\right)}\right](x) .
$$


Proof. See Appendix A.2

Using Corollary 3.6, we can easily derive a stability estimate for the conical Radon transform. For that purpose we denote by $\mathcal{F} f$ the Fourier transform of $f$ and set

$$
\begin{aligned}
\|f\|_{-1}^{2} & =\int_{\mathbb{R}^{3}}|\mathcal{F} f(\xi)|^{2}\left(\|\xi\|^{2}+1\right)^{-1} \mathrm{~d} \xi \\
\left\|\mathcal{C}_{k} f\right\|^{2} & =\int_{0}^{\pi} \int_{\mathbb{R}} \int_{0}^{2 \pi} \int_{0}^{\pi}\left|\frac{\mathcal{C}_{k} f(\varphi, z, \beta, \psi)}{\cos ^{2-k}(\psi)}\right|^{2} \cos (\beta) \sin (\beta) \mathrm{d} \psi \mathrm{d} \varphi \mathrm{d} z \mathrm{~d} \beta \\
\left\|\mathcal{C}_{k} f\right\|_{1}^{2} & =\left\|\mathcal{C}_{k} f\right\|^{2}+\left\|\partial_{z} \mathcal{C}_{k} f\right\|^{2} .
\end{aligned}
$$

Theorem 3.7. For $f \in C_{c}^{\infty}\left(D_{1}(0) \times \mathbb{R}\right)$ we have $\|f\|_{-1} \leq\left\|\mathcal{C}_{1} f\right\|$ and $\|f\| \leq \pi / 2\left\|\mathcal{C}_{0} f\right\|_{1}$.

Proof. By Corollary [3.6 with $k=1$ we have

$$
\begin{aligned}
& \|f\|_{-1}^{2} \leq \int_{\mathbb{R}^{3}}|\mathcal{F} f(\xi)|^{2}\|\xi\|^{-2} \mathrm{~d} \xi \\
& =-(2 \pi)^{3} \int_{\mathbb{R}^{3}} f(x) \Delta_{x}^{-1} f(x) \mathrm{d} x \\
& =\frac{1}{\pi} \int_{\mathbb{R}^{3}} f(x) \mathcal{C}_{1}^{\sharp}\left[\int_{0}^{\pi} \frac{\mathcal{C}_{1} f\left(\varphi, z, \beta, \psi^{\prime}\right) \cos (\beta) \sin (\beta) \mathrm{d} \psi^{\prime}}{\cos (\psi) \cos \left(\psi^{\prime}\right)}\right](x) \mathrm{d} x \\
& =\frac{1}{\pi} \int_{0}^{\pi} \int_{0}^{\pi} \int_{\mathbb{R}} \int_{0}^{2 \pi} \mathcal{C}_{1} f(\varphi, z, \beta, \psi) \int_{0}^{\pi} \frac{\mathcal{C}_{1} f\left(\varphi, z, \beta, \psi^{\prime}\right) \cos (\beta) \sin (\beta) \mathrm{d} \psi^{\prime}}{\cos (\psi) \cos \left(\psi^{\prime}\right)} \mathrm{d} \varphi \mathrm{d} z \mathrm{~d} \beta d \psi \\
& =\frac{1}{\pi} \int_{0}^{\pi} \int_{\mathbb{R}}^{2 \pi}\left(\int_{0}^{\pi} \frac{\mathcal{C}_{1} f(\varphi, z, \beta, \psi)}{\cos (\psi)} \mathrm{d} \psi\right)^{2} \cos (\beta) \sin (\beta) \mathrm{d} \varphi \mathrm{d} z \mathrm{~d} \beta
\end{aligned}
$$

where in the second last equality holds because $\mathcal{C}_{1}^{\sharp}$ is the $L^{2}$-adjoint of $\mathcal{C}_{1}$. Applying Jensen's inequality to the inner integral completes our proof.

Now suppoe $k=0$. Similar to (3.18) we have

$$
\begin{aligned}
\mid f \|^{2}=\frac{1}{\pi} \int_{0}^{\pi} & \int_{\mathbb{R}} \int_{0}^{2 \pi}\left(\int_{0}^{\pi} \frac{\mathcal{C}_{0} f(\varphi, z, \beta, \psi)}{\cos ^{2}(\psi)} \mathrm{d} \psi\right) \\
& \times\left(\int_{0}^{\pi} \frac{\partial_{z} \mathcal{C}_{0} f(\varphi, z, \beta, \psi)}{\cos ^{2}(\psi)} \mathrm{d} \psi\right) \cos (\beta) \sin (\beta) \mathrm{d} \varphi \mathrm{d} z \mathrm{~d} \beta \\
\leq \frac{1}{\pi}\left(\int_{0}^{\pi}\right. & \left.\int_{\mathbb{R}}^{2 \pi} \int_{0}^{2 \pi}\left(\int_{0}^{\pi} \frac{\mathcal{C}_{0} f(\varphi, z, \beta, \psi)}{\cos ^{2}(\psi)} \mathrm{d} \psi\right)^{2} \cos (\beta) \sin (\beta) \mathrm{d} \varphi \mathrm{d} z \mathrm{~d} \beta\right)^{1 / 2} \\
& \times\left(\int_{0}^{\pi} \int_{\mathbb{R}} \int_{0}^{2 \pi}\left(\int_{0}^{\pi} \frac{\partial_{z} \mathcal{C}_{0} f(\varphi, z, \beta, \psi)}{\cos ^{2}(\psi)} \mathrm{d} \psi\right)^{2} \cos (\beta) \sin (\beta) \mathrm{d} \varphi \mathrm{d} z \mathrm{~d} \beta\right)^{1 / 2},
\end{aligned}
$$

where in the second line, we used the Cauchy-Schwarz inequality. By Jensen's inequality,

$$
\|f\|^{2} \leq \pi\left(\int_{0}^{\pi} \int_{\mathbb{R}} \int_{0}^{2 \pi} \int_{0}^{\pi}\left|\frac{\mathcal{C}_{0} f(\varphi, z, \beta, \psi)}{\cos ^{2}(\psi)}\right|^{2} \mathrm{~d} \psi \cos (\beta) \sin (\beta) \mathrm{d} \varphi \mathrm{d} z \mathrm{~d} \beta\right)^{1 / 2}
$$




$$
\begin{gathered}
\times\left(\int_{0}^{\pi} \int_{\mathbb{R}} \int_{0}^{2 \pi} \int_{0}^{\pi}\left|\frac{\partial_{z} \mathcal{C}_{0} f(\varphi, z, \beta, \psi)}{\cos ^{2}(\psi)}\right|^{2} \mathrm{~d} \psi \cos (\beta) \sin (\beta) \mathrm{d} \varphi \mathrm{d} z \mathrm{~d} \beta\right)^{1 / 2} \\
\leq \frac{\pi}{2}\left(\int_{0}^{\pi} \int_{\mathbb{R}}^{2 \pi} \int_{0}^{\pi} \int_{0}\left|\frac{\mathcal{C}_{0} f(\varphi, z, \beta, \psi)}{\cos ^{2}(\psi)}\right|^{2} \mathrm{~d} \psi \cos (\beta) \sin (\beta) \mathrm{d} \varphi \mathrm{d} z \mathrm{~d} \beta\right) \\
+\frac{\pi}{2}\left(\int_{0}^{\pi} \int_{\mathbb{R}} \int_{0}^{2 \pi} \int_{0}^{\pi}\left|\frac{\partial_{z} \mathcal{C}_{0} f(\varphi, z, \beta, \psi)}{\cos ^{2}(\psi)}\right|^{2} \mathrm{~d} \psi \cos (\beta) \sin (\beta) \mathrm{d} \varphi \mathrm{d} z \mathrm{~d} \beta\right) .
\end{gathered}
$$

\section{Conclusion}

In this paper we studied the weighted conical Radon $\mathcal{C}_{k}$ with vertices on the cylinder and presented two explicit reconstruction procedures (see Algorithms 3 and 4). The first approach is based on reducing $\mathcal{C}_{k}$ to the $\mathrm{V}$-line transform with vertices on the circle. For the $\mathrm{V}$-line transform we derived an explicit inversion formula based on Fourier series expansion and a corresponding efficient discrete reconstruction algorithm. We believe that also Algorithms 3 and 4 for inverting $\mathcal{C}_{k}$ can be implemented efficiently. Future work will be done to numerically implement these reconstruction methods. We intend to compare these methods with iterative procedures in terms of computation time and image quality for realistically simulated data.

\section{A Proofs}

\section{A.1 Formal $L^{2}$-adjoint of $\mathcal{C}_{k}$}

We have

$$
\begin{aligned}
& \int_{0}^{\pi} \int_{0}^{\pi} \int_{\mathbb{R}} \int_{0}^{2 \pi} \mathcal{C}_{k} f(\varphi, z, \beta, \psi) g(\varphi, z, \beta, \psi) \mathrm{d} \varphi \mathrm{d} z \mathrm{~d} \beta \mathrm{d} \psi \\
& =\int_{0}^{\pi} \int_{0}^{\pi} \int_{\mathbb{R}} \int_{0}^{2 \pi} \int_{\mathbb{R}^{3}} f((\theta(\varphi), z)+x) \delta(a(\varphi, \beta) \cdot x-\|x\| \cos (\psi)) \\
& \quad \times g(\varphi, z, \beta, \psi) \frac{\sin (\psi) \mathrm{d} x}{\|x\|^{1-k}} \mathrm{~d} \varphi \mathrm{d} z \mathrm{~d} \beta \mathrm{d} \psi \\
& =\int_{\mathbb{R}^{3}} \int_{0}^{\pi} \int_{0}^{\pi} \int_{\mathbb{R}} \int_{0}^{2 \pi} f(x) g(\varphi, z, \beta, \psi)\|x-(\theta(\varphi), z)\|^{k-1} \\
& \quad \times \delta(a(\varphi, \beta) \cdot x-z \cos (\beta)+\sin (\beta)-\|x-(\theta(\varphi), z)\| \cos (\psi)) \sin (\psi) \mathrm{d} \varphi \mathrm{d} z \mathrm{~d} \beta \mathrm{d} \psi \mathrm{d} x \\
& =\int_{\mathbb{R}^{3}} f(x) \mathcal{C}_{k}^{\sharp} g(x) \mathrm{d} x .
\end{aligned}
$$

Here for the first and second equalities, we made use of the change of variables $x \leftarrow r \omega$ and $x \leftarrow(\theta(\varphi), z)+x$, respectively. 


\section{A.2 Proof of Corollary 3.6}

It is enough to show that for a fixed $x \in \mathbb{R}^{3}$ and $k=0,1$, we have

$$
\begin{aligned}
\mathcal{C}_{k}^{\sharp}\left[\int_{0}^{\pi} \frac{\partial_{z}^{1-k} g\left(\varphi, z, \beta, \psi^{\prime}\right) \cos (\beta) \sin (\beta) \mathrm{d} \psi^{\prime}}{\cos ^{2-k}(\psi) \cos ^{2-k}\left(\psi^{\prime}\right)}\right](x) \\
=(-1)^{k+1} \int_{\mathbb{S}^{2}} \int_{\mathbb{R}} \int_{0}^{\pi} \frac{\partial_{z}^{2-2 k} g(\varphi, z, \beta, \psi) \cos ^{k}(\beta) \mathrm{d} \psi \mathrm{d} z \mathrm{~d} S(a(\varphi, \beta))}{(a(\varphi, \beta) \cdot x-z \cos (\beta)+\sin (\beta)) \cos ^{2-k}(\psi)} .
\end{aligned}
$$

By the definition of $\mathcal{C}_{k}^{\sharp}$ and the homogeneity of the one-dimensional delta-distribution, the left hand side of (A.1) becomes

$$
\begin{aligned}
\int_{0}^{\pi} \int_{\mathbb{R}} \int_{0}^{2 \pi} \int_{0}^{\pi} \int_{0}^{\pi} \frac{\partial_{z}^{1-k} g\left(\varphi, z, \beta, \psi^{\prime}\right) \cos (\beta) \sin (\beta)}{\cos ^{2-k}(\psi) \cos ^{2-k}\left(\psi^{\prime}\right)\|x-(\theta(\varphi), z)\|^{2-k}} \\
\quad \times \delta\left(\frac{a(\varphi, \beta) \cdot x-z \cos (\beta)+\sin (\beta)}{\|x-(\theta(\varphi), z)\|}-\cos (\psi)\right) \sin (\psi) \mathrm{d} \psi^{\prime} \mathrm{d} \psi \mathrm{d} \varphi \mathrm{d} z \mathrm{~d} \beta \\
=\int_{0}^{\pi} \int_{\mathbb{R}} \int_{0}^{2 \pi} \int_{0}^{\pi} \frac{\partial_{z}^{1-k} g\left(\varphi, z, \beta, \psi^{\prime}\right) \cos (\beta) \sin (\beta) \mathrm{d} \psi^{\prime} \mathrm{d} \varphi \mathrm{d} z \mathrm{~d} \beta}{(a(\varphi, \beta) \cdot x-z \cos (\beta)+\sin (\beta))^{2-k} \cos ^{2-k}\left(\psi^{\prime}\right)} .
\end{aligned}
$$

Now the surface measure of the sphere is $\sin (\beta) \mathrm{d} \varphi \mathrm{d} \beta$ when $\varphi$ and $\beta$ are the azimuthal and polar angles, respectively. Thus we have

$$
\begin{aligned}
\mathcal{C}_{k}^{\sharp}\left[\int_{0}^{\pi} \frac{\partial_{z}^{1-k} g\left(\varphi, z, \beta, \psi^{\prime}\right) \cos (\beta) \sin (\beta) \mathrm{d} \psi^{\prime}}{\cos ^{2-k}(\psi) \cos ^{2-k}\left(\psi^{\prime}\right)}\right](x) \\
\quad=\int_{\mathbb{S}^{2}} \int_{\mathbb{R}} \int_{0}^{\pi} \frac{\partial_{z}^{1-k} g(\varphi, z, \beta, \psi) \cos (\beta) \mathrm{d} \psi \mathrm{d} z \mathrm{~d} S(\varphi, \beta)}{(a(\varphi, \beta) \cdot x-z \cos (\beta)+\sin (\beta))^{2-k} \cos ^{2-k}(\psi)} .
\end{aligned}
$$

If $k=1$, the proof is done. If $k=0$, then the integration by parts completes the proof.

\section{B Generalization to higher dimension}

In this section we generalize the results for the conical Radon transform presented in Subsection 3.2 to a general dimension. For the following let $n \geq 3$.

Definition B.1 (The conical Radon transform on the cylinder in $\left.\mathbb{R}^{n}\right)$. Let $f \in C_{c}^{\infty}\left(B_{1}(0) \times\right.$ $\mathbb{R})$. We define the conical Radon transform in $\mathbb{R}^{n} \mathcal{C} f: \mathbb{S}^{n-2} \times \mathbb{R} \times(0, \pi)^{2} \rightarrow \mathbb{R}$ by

$$
\mathcal{C} f(\theta, z, \beta, \psi):=\sin (\psi) \int_{\mathbb{S}^{n-1}} \int_{0}^{\infty} f((\theta, z)+r \omega) r^{n-2} \delta(\omega \cdot a(\theta, \beta)-\cos (\psi)) \mathrm{d} r \mathrm{~d} S(\omega),
$$

where $a(\theta, \beta):=(-\theta \sin (\beta), \cos (\beta))$.

As in the three-dimensional case the formal $L^{2}$-adjoint of $\mathcal{C}$ is given by

$$
\begin{aligned}
\mathcal{C}^{\sharp} g(x) & =\int_{0}^{\pi} \int_{0}^{\pi} \int_{\mathbb{R}} \int_{\mathbb{S}^{n-2}} g(\theta, z, \beta, \psi) \\
& \times \delta(x \cdot a(\theta, \beta)-z \cos (\beta)+\sin (\beta)-\|x-(\theta, z)\| \cos (\psi)) \sin (\psi) \mathrm{d} S(\theta) \mathrm{d} z \mathrm{~d} \beta \mathrm{d} \psi .
\end{aligned}
$$

We further use the following notations: 
* $\mathcal{R} f(\omega, s)$ for the regular $n$-dimensional Radon transform;

* $\mathcal{F} f(\xi)$ for the $n$-dimensional Fourier transform;

* $\left(-\Delta_{x}\right)^{(n-1) / 2} f:=\mathcal{F}^{-1}\left(\|\xi\|^{n-1} \mathcal{F} f\right)$ for the fractional Laplacian;

* $\|f\|_{-(n-1) / 2}^{2}:=\int_{\mathbb{R}^{n}}|\mathcal{F} f(\xi)|^{2}\left(\|\xi\|^{2}+1\right)^{-(n-1) / 2} \mathrm{~d} \xi ;$

* $\|\mathcal{C} f\|^{2}:=\int_{0}^{\pi} \int_{\mathbb{R}} \int_{\mathbb{S}^{n-2}} \int_{0}^{\pi}|\mathcal{C} f(\theta, z, \beta, \psi) / \cos (\psi)|^{2} \cos (\beta) \sin (\beta) \mathrm{d} \psi \mathrm{d} S(\theta) \mathrm{d} z \mathrm{~d} \beta$.

Similar to the three-dimensional case one then has the following results.

Theorem B.2. For any $f \in C_{c}^{\infty}\left(B_{1}(0) \times \mathbb{R}\right)$ the following hold:

Relation to Radon transform: For every $(\theta, \beta, t) \in \mathbb{S}^{n-1} \times(0, \pi) \times \mathbb{R}$,

$$
\mathcal{R} f(a(\theta, \beta), t)=\frac{1}{\pi^{2}} \int_{\mathbb{R}} \int_{0}^{\pi} \frac{\mathcal{C} f(\theta, z, \beta, \psi) \cos (\beta) \mathrm{d} \psi \mathrm{d} z}{(t-z \cos (\beta)+\sin (\beta)) \cos (\psi)} .
$$

INVERSION FORMULAS: For every $x \in \mathbb{R}^{n}$,

$$
\begin{aligned}
& f(x)=\frac{1}{\pi(2 \pi)^{n}} \Delta_{x}^{(n-1) / 2} \int_{0}^{\pi} \int_{\mathbb{S}^{n-2}} \int_{\mathbb{R}} \int_{0}^{\pi} \frac{\mathcal{C} f(\theta, z, \beta, \psi) \cos (\beta) \sin (\beta) \mathrm{d} \psi \mathrm{d} z \mathrm{~d} S(\theta) \mathrm{d} \beta}{(x \cdot a(\theta, \beta)-z \cos (\beta)+\sin (\beta)) \cos (\psi)}, \\
& f(x)=\frac{1}{\pi(2 \pi)^{n}} \Delta_{x}^{(n-1) / 2} \mathcal{C}^{\sharp}\left[\int_{0}^{\pi} \frac{\mathcal{C} f\left(\theta, z, \beta, \psi^{\prime}\right) \cos (\beta) \sin (\beta) \mathrm{d} \psi^{\prime}}{\cos (\psi) \cos \left(\psi^{\prime}\right)}\right](x) .
\end{aligned}
$$

Stability estimate: $\|f\|_{-(n-1) / 2} \leq\|\mathcal{C} f\|$.

Proof. The proof is analogous to the three-dimensional case and is therefore omitted.

Similar to the three-dimensional case one can derive inversion formulas and stability estimates for the weighted conical Radon transform.

\section{References}

[1] M. Allmaras, D. Darrow, Y. Hristova, G. Kanschat, and P. Kuchment, Detecting small low emission radiating sources, Inverse Probl. Imaging, 7 (2013), pp. 4779 .

[2] R. Basko, G. L. Zeng, and G. T. Gullberg, Analytical reconstruction formula for one-dimensional Compton camera, IEEE Trans. Nucl. Sci., 44 (1997), pp. 1342-1346.

[3] R. Basko, G. L. Zeng, and G. T. Gullberg, Application of spherical harmonics to image reconstruction for the Compton camera, Phys. Med. Biol., 43 (1998), p. 887.

[4] A. M. Conmack, Representation of a function by its line integrals, with some radiological applications, J. Appl. Phys., 34 (1963), pp. 2722-2727.

[5] M. J. CRee And P. J. Bones, Towards direct reconstruction from a gamma camera based on Compton scattering, IEEE Trans. Med. Imag., 13 (1994), pp. 398-407. 
[6] D. B. Everett, J. S. Fleming, R. W. Todd, And J. M. Nightingale, Gammaradiation imaging system based on the Compton effect, Proc. IEEE, 124 (1977), pp. 9951000.

[7] L. Florescu, J. C. Schotland, and V. A. Markel, Single-scattering optical tomography, Phys. Rev. E, 79 (2009), p. 036607.

[8] R. Gouia-Zarrad and G. Ambartsoumian, Exact inversion of the conical Radon transform with a fixed opening angle, Inverse Probl., 30 (2014), pp. 045007, 12.

[9] M. Haltmeier, Exact reconstruction formulas for a Radon transform over cones, Inverse Probl., 30 (2014).

[10] E. W. Hansen, Circular harmonic image reconstruction: experiments, Appl. Opt., 20 (1981), pp. 2266-2274.

[11] S. Helgason, The Radon Transform, vol. 5 of Progress in Mathematics, Birkhäuser, Boston, second ed., 1999.

[12] C. Jung And S. Moon, Inversion formulas for cone transforms arising in application of Compton cameras, Inverse Probl., 31 (2015), pp. 015006, 20.

[13] C. Jung AND S. Moon, Exact inversion of the cone transform arising in an application of a Compton camera consisting of line detectors, SIAM J. Iimaging Scinces, 9 (2016), pp. 520-536.

[14] S. Moon, On the determination of a function from its conical Radon transform with a fixed central axis, SIAM J. Math. Anal., 48 (2016), pp. 1833-1847.

[15] M. Morvidone, M. K. Nguyen, T. T. Truong, and H. Zaidi, On the V-line Radon transform and its imaging applications, Int. J. Biomed. Imaging, 2010 (2010), p. 11.

[16] F. Natterer, The Mathematics of Computerized Tomography, vol. 32 of Classics in Applied Mathematics, SIAM, Philadelphia, 2001.

[17] R. M. PERRY, Reconstructing a function by circular harmonic analysis of line integrals, in Image Processing for 2-D and 3-D Reconstruction from Projections: Theory and Practice in Medicine and the Physical Sciences, Digest of technical papers, Stanford, California, 1975.

[18] D. Schiefeneder And M. Haltmeier, The Radon transform over cones with vertices on the sphere and orthogonal axes. http://arxiv.org/abs/1606.03486, 2016.

[19] M. Singh, An electronically collimated gamma camera for single photon emission computed tomography. part I: Theoretical considerations and design criteria, Med. Phys., 10 (1983), p. 1983. 
[20] B. Smith, Reconstruction methods and completeness conditions for two Compton data models, J. Opt. Soc. Am. A, 22 (2005), pp. 445-459.

[21] B. Smith, Line-reconstruction from Compton cameras: data sets and a camera design, Opt. Eng., 50 (2011), p. 053204.

[22] F. Terzioglu, Some inversion formulas for the cone transform, Inverse Probl., 31 (2015), pp. 115010, 21.

[23] R. W. Todd, J. M. Nightingale, And D. B. Everett, A proposed gamma camera, Nature, 251 (1974), pp. 132-134.

[24] T. T. Truong and M. K. Nguyen, On new V-line Radon transforms in $\mathbb{R}^{2}$ and their inversion, J. Phys. A, 44 (2011), pp. 075206, 13.

[25] M. WERnick And J. N. AARsvold, Emission tomography: the fundamentals of PET and SPECT, Academic Press, 2004. 